INPLASY

PROTOCOL

To cite: Jiang et al. Chinese herbal medicine for immune infertility: A protocol for systematic review and metaanalysis. Inplasy protocol 2020120073. doi:

10.37766/inplasy2020.12.0073

Received: 12 December 2020

Published: 12 December 2020

Corresponding author:

Ying Ye

yeying@cdutcm.edu.cn

Author Affiliation:

School of Basic Medical

Sciences, Chengdu University

of Traditional Chinese Medicine

Support: NSFC Grant

No.61801058.

Review Stage at time of this submission: Preliminary

searches.

Conflicts of interest:

None.

\section{Chinese herbal medicine for immune infertility: A protocol for systematic review and meta-analysis}

Review question / Objective: The purpose of this study is to evaluate the efficacy and safety of traditional Chinese medicine for the treatment of immune infertility.

Condition being studied: Infertility is a reproductive disorder caused by multiple causes and is an adverse event of reproductive health for couples in the reproductive period. Women who do not avoid sex for at least 12 months and are not pregnant are said to be infertile. $10 \% \sim 20 \%$ of infertility is caused by immune factors. At present, there is no unified diagnostic standard for immunological infertility. Clinically, it is considered that abnormal ovulation and reproductive system function of women are excluded, and no obvious pathogenic factors occur; routine examination of male semen is normal, but there is evidence of anti-reproductive immunity, thus causing infertility is immunological infertility. Traditional Chinese medicine (TCM) has a long history of treating infertility and has remarkable curative effect. It plays an important role in the treatment of gynecological and obstetrical diseases in China. The purpose of this study is to evaluate the efficacy and safety of traditional Chinese medicine for the treatment of immune infertility.

INPLASY registration number: This protocol was registered with the International Platform of Registered Systematic Review and Meta-Analysis Protocols (INPLASY) on 12 December 2020 and was last updated on 12 December 2020 (registration number INPLASY2020120073).

\section{INTRODUCTION}

Review question / Objective: The purpose of this study is to evaluate the efficacy and safety of traditional Chinese medicine for the treatment of immune infertility.
Condition being studied: Infertility is a reproductive disorder caused by multiple causes and is an adverse event of reproductive health for couples in the reproductive period. Women who do not avoid sex for at least 12 months and are not pregnant are said to be infertile. $10 \%$ 
$20 \%$ of infertility is caused by immune factors. At present, there is no unified diagnostic standard for immunological infertility. Clinically, it is considered that abnormal ovulation and reproductive system function of women are excluded, and no obvious pathogenic factors occur; routine examination of male semen is normal, but there is evidence of antireproductive immunity, thus causing infertility is immunological infertility. Traditional Chinese medicine (TCM) has a long history of treating infertility and has remarkable curative effect. It plays an important role in the treatment of gynecological and obstetrical diseases in China. The purpose of this study is to evaluate the efficacy and safety of traditional Chinese medicine for the treatment of immune infertility.

\section{METHODS}

Participant or population: All the patients who have been diagnosed with immune infertility will be included. There are no restrictions on age, region, nation, belief, ethnicity, sources, and courses of disease.

Intervention: The experimental group is treated with TCM on the basis of conventional western medicine treatment in the control group. The use of TCM is limited to prescription and Chinese patent medicines. Prescription drugs require a clear dose, but there are no restrictions on the composition, dosage form and dosage. For the dosage, such as decoctions, granules, pills, powders, etc. Other types of TCM treatments such as TCM injections, acupuncture, moxibustion, massage, cupping, and others will be excluded.

Comparator: The control group received routine western medicine treatment, prednisone plus aspirin.

Study designs to be included: We will search all the studies that TCM is used as the main intervention for immune infertility, Non-RCTs quasi-RCTs, series of case reports, and cross research will be excluded. Full article not available will be excluded. No language restrictions.
Eligibility criteria: 1 . Types of studies. We will search all the studies that TCM is used as the main intervention for immune infertility, Non-RCTs quasi-RCTs, series of case reports, and cross research will be excluded. Full article not available will be excluded. No language restrictions. 2. Types of participants. All the patients who have been diagnosed with immune infertility will be included. There are no restrictions on age, region, nation, belief, ethnicity, sources, and courses of disease. 3. Types of interventions. There is no requirement for the ntervention course, the specific contents of the control group and the experimental group are as follows. 3.1. Control intervention. The control group received routine western medicine treatment, prednisone plus aspirin. 3.2. Experimental interventional. The experimental group is treated with TCM on the basis of conventional western medicine treatment in the control group. The use of TCM is limited to prescription and Chinese patent medicines. Prescription drugs require a clear dose, but there are no restrictions on the composition, dosage form and dosage. For the dosage, such as decoctions, granules, pills, powders, etc. Other types of TCM treatments such as TCM injections, acupuncture, moxibustion, massage, cupping, and others will be excluded.

Information sources: The databases of PubMed, CENTRAL (The Cochrane Central Register of Controlled Trials), Excerpt Medica Database (Embase), China National Knowledge Infrastructure (CNKI), Weipu Information Chinese Periodical Service Platform (VIP), Wanfang Data Knowledge Service Platform (WANFANG Data), and China Biomedical Literature Service System (SinoMed) will be searched online. The search time is set from the establishment of the search database to December 2020. According to the standards of the Cochrane Collaboration workbook of the International EvidenceBased Medicine Center, the search terms include "Chinese medicine," "Traditional Chinese medicine," "proprietary Chinese medicine," "Chinese herbal medicine," "immune infertility.". 
Main outcome(s): The primary outcomes are pregnancy rates, the efficiency of Chinese herbal medicine (at least one negative antibody for infertility) and safety index.

Quality assessment / Risk of bias analysis: As for the risk of bias in the literature, two researchers will independently use the tool for assessing risk of bias recommended by Cochrane Handbook for Systematic Reviews of Interventions 5.1.0 (Cochrane Handbook 5.1.0-Part 2: 8.5-8.7) to assess the quality of the included literature and risk of bias. Evaluation content includes: selection bias (random sequence generation, and allocation concealment), performance bias (blinding of participants and personnel), detection bias (blinding of outcome assessment), attrition bias (incomplete outcome data), reporting bias (selective outcome reporting), and other bias (other sources of bias). Evaluators judge the risk level by carefully reading the full text, which is divided into low risk, high risk and unknown risk. If the research reported in the literature is not detailed enough, the judgement is usually "unknown risk" of bias. For example, the study uses random number table for grouping, so the random sequence generation will be expressed as "low risk." If there are any differences, we would consult the third reviewer for solution.

Strategy of data synthesis: The metaanalysis in this review will use RevMan 5.3 and Stata 13.0 software. For the outcome index of the two categorical variables, relative risk (Relativisk, $R R$ ) will be adopted, and for the outcome index of continuous variables, the mean difference (MD) or standardized mean difference (SMD) will be adopted will a confidence interval $(\mathrm{Cl})$ of $95 \%$. Heterogeneity tests will be used for the included studies which will be tested by chi-square test. If $P \geq .10$ and $12 \leq 50 \%$, there is no significant statistical heterogeneity or no statistical difference in heterogeneity, a fixed effect model will be adopted. If $\mathrm{P} 50 \%$, there is significant heterogeneity between studies, a random effect model will be adopted. Further analysis of the source of heterogeneity, if necessary, perform subgroup analysis. There are clinical and methodological differences in the experimental studies. Therefore, random effects models will be selected in this study. Finally, a funnel chart will be drawn to evaluate the publication bias of the literature.

Subgroup analysis: If $\mathrm{P} 50 \%$, there is significant heterogeneity between studies, a random effect model will be adopted. Further analysis of the source of heterogeneity, if necessary, perform subgroup analysis. There are clinical and methodological differences in the experimental studies. Therefore, random effects models will be selected in this study. Finally, a funnel chart will be drawn to evaluate the publication bias of the literature.

Sensibility analysis: Sensitivity analysis will be performed to test the robustness of findings if there are sufficient studies included. we will conduct sensitivity analysis by excluding (1) studies with high risks of bias and (2) outliers that are numerically distant from the rest of the data.

Country(ies) involved: China.

Keywords: immune infertility, Effectiveness, Safety, Chinese herbal medicine, protocol, meta-analysis.

Contributions of each author:

Author 1 - Cui Jiang.

Author 2 - Zhaodi Wang.

Author 3 - Shiqing Yuan.

Author 4 - Yong Jiang.

Author 5 - Ying Ye. 